\title{
Capacidades, hábitos y carácter: atribuciones docentes sobre el alumnado de Bachillerato y Formación Profesional
}

\author{
Aina Tarabini \\ Universitat Autònoma de Barcelona. Facultat de Ciències Polítiques i Sociologia. \\ Departament de Sociologia \\ aina.tarabini@uab.cat
}

\author{
Alba Castejón \\ Universitat Autònoma de Barcelona. Facultat d'Educació. Departament de Pedagogia \\ Sistemàtica i Social \\ alba.castejón@uab.cat

\section{Marta Curran} \\ Universidad Complutense de Madrid. Facultad de Educación y Formación del Profesorado. \\ Departamento de Sociología Aplicada \\ mcurran@ucm.es
}

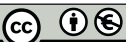

Recepción: 18-10-2019

Aceptación: 27-12-2019

Publicación: 01-04-2020

\section{Resumen}

La división de los sistemas educativos en itinerarios diferenciados — académico y profesional- es uno de los elementos centrales para explicar las pautas de estratificación y desigualdad social en el ámbito internacional. Entre los múltiples factores que dan cuenta de la relación entre itinerarios educativos y desigualdad social, la literatura especializada ha señalado el rol que juegan las expectativas docentes como factor crucial para entender la asignación de diferentes estudiantes a distintos itinerarios. En el contexto español, es todavía escasa la literatura que aborda el rol de las creencias docentes para entender las pautas de desigualdad social asociadas con las transiciones educativas de los y las jóvenes. En este contexto, el objetivo del artículo es analizar la imagen que el profesorado tiene construida del alumnado de Bachillerato y de Formación Profesional e investigar las características atribuidas a los y las estudiantes de ambos itinerarios formativos en términos de capacidades, hábitos y carácter. Partiendo de una perspectiva constructivista, el análisis se basa en 37 entrevistas en profundidad con tutores/as de ESO y Bachillerato y Formación Profesional de 8 centros educativos de la ciudad de Barcelona. Los resultados muestran una concepción ampliamente dicotómica del alumnado de Bachillerato y de FP. Estas concepciones juegan un rol clave en la legitimación discursiva de un sistema de educación posobligatoria altamente segmentado y desigual.

Palabras clave: profesorado; expectativas docentes; itinerarios educativos; transiciones educativas; discurso docente; desigualdad educativa 
Abstract. Ability, manners and character: teachers' attributions on Baccalaureate and VET students

The division of the educational systems into different tracks — academic and VET - represents one of the key elements in explaining social stratification and inequalities. Among the multiple factors that explain the relation between education tracks and social inequality, teachers' expectations are conceived as a crucial element in understanding the distribution of different students in each track. In the Spanish context there is still a lack of literature analyzing the role of teachers' expectations to explain the social inequalities associated to educational transitions. In this context, the aim of the paper is to explore teachers' conceptions of Baccalaureate and VET students, exploring the features attributed to students in each track in terms of abilites, manners and character. Based on a constructivist approach, the article draws on data from a qualitative study based on 37 in-depth interviews to tutors from both secondary compulsory education, Baccalaureate and VET in a sample of 8 secondary schools in the city of Barcelona. The findings of the paper show a remarkably dichotomized conception between students from the academic and the professional track. These conceptions play a key role in the discursive legitimation in a highly segmented and unequal post compulsory education system.

Keywords: teachers; teachers' expectations; educational tracks; educational transitions; teachers' discourses; educational inequalities

\section{Sumario}

\section{Introducción}

2. Fundamentos teóricos: la construcción escolar de la inteligencia y la capacidad 3. Metodología, muestra y procedimientos de análisis
4. Resultados: concepciones docentes sobre el alumnado

5. Conclusiones

Referencias bibliográficas

\section{Introducción ${ }^{1}$}

La división de los sistemas educativos en itinerarios diferenciados ha sido señalada por la literatura internacional como un mecanismo clave de reproducción de la desigualdad social (Tarabini e Ingram, 2018). De hecho, los puntos de inflexión dentro de los sistemas educativos son un elemento crucial para entender el grado de estratificación y selectividad social de diferentes países en términos de clase, género y etnicidad (Bernardi y Requena, 2010; Blossfeld y Shavit, 2011). La relación entre itinerarios educativos y desigualdad social, sin embargo, no es lineal, ni tiene el mismo significado e impactos en diferentes países. Autores como Pfeffer (2008) y Shavit y Muller (2000) afirman que las variaciones institucionales entre diferentes países — a saber: la oferta de diferen-

1. Este estudio ha sido financiado en el marco del proyecto I+D+I 'EDUPOST16. La construcción de las oportunidades educativas post-16. Un análisis de las transiciones a la educación secundaria posobligatoria en contextos urbanos (Ref. CSO2016-80004P. IP Aina Tarabini). Más información: <http://edupost16.es/>. 
tes itinerarios, las barreras para acceder a los mismos, las formas de certificación y acreditación, etcétera - son el elemento central para explicar el grado de selectividad asociado con las transiciones educativas. En otro lado (Tarabini y Jacovkis, 2020, en prensa) nos hemos ocupado de señalar cómo operan estos elementos en el caso español, cómo son interpretados por diferentes agentes políticos y escolares y qué impactos tienen en términos de oportunidades sociales. El foco del presente artículo, sin embargo, es otro: abordar el rol que tiene el profesorado como agente clave para generar diferentes oportunidades de transición educativa entre los y las jóvenes.

La literatura internacional ha señalado, en particular, el rol central que juegan las expectativas docentes para explicar la asignación de los y las estudiantes a diferentes itinerarios educativos (Bonizzoni et al., 2016; Lappalainen et al., 2012; Houtte et al., 2013). Dichas expectativas, transmitidas a través del trato, tienen impactos en la autoestima (Agirdag et al., 2012), la actitud (Demanet y Houtte, 2012) y los resultados de los y las estudiantes (Bergh et al., 2010), y, en términos globales, contribuyen a explicar por qué los itinerarios profesionales tienden a estar sobrerrepresentados por chicos de bajo estatus socioeconómico y cultural, de origen migrado o de grupos étnicos minoritarios.

En el contexto español, es numerosa la investigación que analiza el rol de las expectativas docentes en las oportunidades educativas de los y las jóvenes (ver, por ejemplo, el monográfico de la RASE, 2015, vol. 8, núm. 3, sobre «Procesos de etiquetaje y escuela»). Es especialmente significativa la literatura que apunta a la relación entre expectativas docentes y agrupación del alumnado dentro de la Educación Secundaria Obligatoria (ESO) (Castejón, 2017; Pàmies, 2013). Dicha literatura señala, del mismo modo que la investigación internacional, que las creencias sesgadas sobre las "capacidades» $\mathrm{y}$ «habilidades» de los y las estudiantes tienen un papel central para explicar qué tipo de alumnos están escolarizados en los grupos de "mayor o menor nivel académico» dentro de los institutos. A pesar de ello, en el contexto español, es todavía escasa la literatura que aborda el rol de las creencias docentes para entender las pautas de desigualdad social asociadas con las transiciones educativas de los y las jóvenes, especialmente en el paso de la educación obligatoria a la posobligatoria, que es cuando el sistema educativo español se divide por primera vez en dos itinerarios diferenciados: el académico (Bachillerato) y el profesional (FP). ${ }^{2}$

En este contexto, el objetivo del artículo es analizar la imagen que el profesorado tiene construida del alumnado de Bachillerato y de FP e investigar concretamente las características atribuidas a los y las estudiantes de ambos itinerarios formativos en términos de capacidades, hábitos y carácter. Este tipo de análisis es especialmente significativo en el contexto español porque se trata

2. El sistema educativo español es obligatorio y formalmente comprensivo desde los 6 hasta los 16 años (6-12, Educación Primaria; 12-16, Educación Secundaria Obligatoria, ESO). Una vez acabada la ESO, el sistema educativo se divide en dos itinerarios de educación secundaria posobligatoria, el académico (Bachillerato) y el profesional (Grados Medios de Formación Profesional, GMFP), y, por tanto, este es el primer momento en el que los y las jóvenes pueden elegir entre diferentes opciones formativas. 
de uno de los sistemas educativos de toda la Unión Europea con mayor polarización de su estructura formativa, hecho que se explica fundamentalmente por el bajo prestigio y el escaso desarrollo histórico que ha tenido la FP (García y Lorente, 2015). Asimismo, todos los datos existentes en este campo señalan la existencia de una fuerte segregación social en el tipo de alumnado que cursa el itinerario académico y el profesional (INE, 2019; MEFP, 2018). Algunas de las preguntas que orientan el análisis son las siguientes: ¿de qué manera y cómo explica el profesorado el perfil del alumnado de Bachillerato y FP?, ¿cómo se describen las características cognitivas, conductuales y personales del alumnado de ambos itinerarios?, ¿qué diferencias y similitudes se identifican en los perfiles de los y las estudiantes de las dos vías formativas?, ¿qué conexiones se hacen entre el perfil de los y las estudiantes y las características de cada itinerario?

El artículo se estructura en cuatro apartados que siguen a esta introducción. En el primer apartado se presenta el marco teórico del artículo, centrado en la producción escolar de las capacidades y en sus impactos en clave de desigualdad social. En el segundo se explica la metodología del estudio, con la descripción de los métodos de recogida y análisis de la información. El tercer apartado presenta el análisis en profundidad de los resultados. El último, a modo de conclusión, muestra una reflexión global sobre las relaciones entre transiciones educativas, discursos docentes y producción y reproducción de la desigualdad social.

\section{Fundamentos teóricos: la construcción escolar de la inteligencia y la capacidad}

La aproximación teórica de este artículo se enmarca en la tradición constructivista sobre la inteligencia y las capacidades desarrollada desde las ciencias sociales y, en particular, desde la sociología de la educación. Específicamente, nos centramos en aquellos estudios que abordan los discursos docentes sobre la capacidad de sus estudiantes a partir del análisis en profundidad de las expectativas docentes y de sus impactos en términos de prácticas pedagógicas (Gamoran, 2009; Oakes et al., 1997), y la configuración de las identidades académicas y sociales de los y las jóvenes (Youdell, 2006).

Desde mediados de los años ochenta del siglo pasado, la psicología cognitiva y del desarrollo propuso nuevas concepciones sobre la inteligencia que contribuyeron a cuestionar la naturaleza biológica, fija y unidimensional que se le había atribuido hasta el momento desde las teorías psicológicas dominantes (Gardner, 1988; Sternberg y Davidson, 1986). Desde este paradigma, se entiende que la inteligencia es siempre contextual y, por tanto, producto de la experiencia y de las interacciones sociales que se van generando a lo largo del tiempo. Como señala Sternberg (2007), la «inteligencia» se concibe de formas muy dispares en diferentes contextos culturales, de tal modo que aspectos como la competencia social, la habilidad cognitiva o la capacidad de resolución de problemas juegan un papel muy diferente en las concepciones de inteligencia existentes en diferentes países, momentos históricos e instituciones sociales. 
El estudio de la inteligencia y de sus implicaciones en términos de desigualdades sociales y educativas no ha sido dominante dentro de la sociología de la educación (Hatt, 2007; 2012; Nash, 2005). De hecho, como señala Hatt (2012), la mayor parte de la investigación sobre esta cuestión sigue estando enmarcada dentro de las fronteras disciplinares de la psicología y, por tanto, no se tiende a contemplar la articulación entre las concepciones de inteligencia y las relaciones de poder, estatus y desigualdad social que cruzan la organización social y la organización escolar en particular. Así, y citando a Kincheloe, Steinberg y Villaverde (1999, en Hatt, 2012), la autora defiende la necesidad de repensar la concepción de «inteligencia» dentro de la institución escolar poniendo de manifiesto sus conexiones con la construcción social del conocimiento escolar y sus implicaciones en términos de las posibilidades de éxito escolar de diferentes estudiantes.

La sociología de la educación, de hecho, ha producido, desde los años ochenta y en el marco de la nueva sociología de la educación (NSE), numerosas investigaciones centradas precisamente en estudiar cómo la organización y la transmisión del conocimiento escolar reflejan las relaciones de poder y desigualdad social (Anyon, 1981; Connell, 1997; Young, 1971). En este sentido, tal como señalan Jennie Oakes et al. (1997), la sociología del conocimiento aporta tres grandes aprendizajes para el estudio de la «inteligencia» y la "capacidad» en el ámbito escolar: 1) el conocimiento es siempre socialmente construido y no un hecho científico objetivo; 2) las concepciones sobre la inteligencia son socialmente construidas más que científicamente descubiertas; y 3) las respuestas escolares a las diferencias de inteligencia (estructuras y prácticas escolares) son también construcciones sociales, y no implicaciones evidentes de formas establecidas de conocimiento científico.

Los estudios etnográficos desarrollados por Oakes $(1982 ; 1985)$ en diversas escuelas de Estados Unidos durante los años ochenta y noventa mostraron claramente que las definiciones sobre la inteligencia son siempre sensibles a los contextos culturales donde se producen y que las concepciones docentes sobre la misma tienen un papel central para explicar la reproducción de las desigualdades educativas. Oakes et al. (1997), de hecho, acuñaron el concepto de «ideología de la inteligencia» para referirse a la forma a través de la cual los discursos docentes mayoritarios sobre la «inteligencia», la "habilidad» y la «capacidad» de sus estudiantes tendían a construirse de modo aislado de sus contextos sociales de referencia, lo que servía para mantener y legitimar unas relaciones de poder sistemáticamente asimétricas entre grupos sociales.

De forma más reciente, Hatt (2012) propone referirse a la inteligencia como una "práctica cultural» y no exclusivamente como una «ideología», ya que, según la autora, el uso del concepto «inteligencia» dentro de las escuelas no funciona solo como una «ideología» o una creencia explícita, sino sobre todo como una práctica basada en teorías implícitas sobre qué significa ser «listo/a» en el ámbito escolar. La «inteligencia», según afirma la autora, opera dentro de las escuelas más como un verbo que como un nombre. Es decir, «es algo que se hace a los otros como forma de posicionamiento social. Y es también una 
herramienta para determinar la identidad de los otros y dotar de sentido a la propia identidad» (2012: 439).

Sea como ideología o como práctica, de forma explícita o implícita, tanto las investigaciones de Oakes et al. (1997) y Hatt (2012) como las de otras autoras que han desarrollado sus análisis en este campo (Gillborn y Youdell, 2001; Hamilton, 2006; Korp, 2011; etcétera) coinciden en señalar cuatro grandes aspectos que se derivan del análisis de las concepciones docentes sobre la inteligencia y sus impactos en términos de desigualdad: 1) el tipo de teorías sobre la inteligencia y la capacidad dominante entre los y las docentes; 2) la relación entre estas concepciones y las expectativas sesgadas en términos de género, origen social y etnicidad; 3) las formas a partir de las cuales dichas teorías y concepciones son institucionalizadas a través de diferentes prácticas y dispositivos pedagógicos; y 4) sus impactos en clave de construcción de identidades de los y las estudiantes. ${ }^{3}$ A continuación, se revisan las principales aportaciones de la literatura en cada uno de estos ámbitos, así como sus implicaciones para el estudio de las transiciones educativas y la reproducción de la desigualdad social.

En primer lugar, y por lo que se refiere a las concepciones docentes sobre la inteligencia y la capacidad, la mayor parte de investigaciones revisadas coinciden en señalar que, de forma mayoritaria, los y las docentes tienden a movilizar teorías implícitas sobre la inteligencia que la conciben como si fuera algo estable, mayormente unidimensional y fácilmente objetivable. Así, por ejemplo, Gillborn y Youdell (2001) afirman que si bien el concepto de inteligencia ha perdido centralidad en los discursos docentes por su tendencia a asociarse con teorías biologicistas, este ha sido reemplazado por un nuevo discurso sobre la habilidad o la capacidad (el nuevo IQism, tal y como lo denominan las autoras) que, si bien goza de más respeto y aceptación social, tiene los mismos impactos en términos de desigualdad social, ya que se adopta de forma eminentemente acrítica y descontextualizada. Tal como afirman las autoras (Gillborn y Youdell, 2001), parece que la habilidad es algo que existe de forma objetiva y dada por descontado, tanto en los discursos docentes como en las políticas educativas, y, por tanto, no es necesario discutir sobre su significado e implicaciones. Siguiendo el mismo argumento, Nash (2005) afirma que, si bien los clásicos conceptos vinculados a la inteligencia y el coeficiente intelectual (IQ) de los y las estudiantes son cada vez más criticados dentro del ámbito escolar, los docentes continúan manteniendo prácticas que garantizan que sus ideas dadas por descontado sobre la distribución social de la habilidad y la capacidad se materialicen en diferentes patrones de resultados académicos. Lejos de culpar al profesorado por dichas concepciones, las investigaciones desarrolladas en este campo apuntan a los efectos que tienen las políticas de reforma educativa sobre las prácticas docentes y sobre la propia definición y articulación de su identidad profesional.

3. Si bien este es uno de los temas centrales abordados en la literatura y por ello se recoge en este apartado, el análisis empírico de este artículo se basa en las concepciones docentes y, por tanto, no se incluyen los impactos en términos de identidades de los estudiantes, que serán abordados en futuras publicaciones sobre este tema. 
Asimismo, es importante señalar el resultado de investigaciones que apuntan a la existencia de una mayor heterogeneidad sobre el significado del concepto capacidad entre el cuerpo docente, sea por las características de diferentes centros educativos, las particularidades de distintos contextos de aprendizaje o las características de diversas áreas de conocimiento. Así, a través de una etnografía sobre un programa de formación profesional de transporte y vehículos en Suecia, Korp (2011) muestra que el significado de lo que se entiende como «ser listo» es negociado en el día a día de las prácticas docentes. En particular, la autora muestra que diferentes contextos instructivos dentro del mismo programa, fundamentalmente talleres o clases convencionales, generan formas distintas de entender la inteligencia por parte del profesorado. Asimismo, la investigación de Jonsson et al. (2012), basada en una muestra de 226 profesores de cuatro escuelas suecas de educación secundaria superior, revela que las concepciones sobre la inteligencia difieren notablemente en función del área de conocimiento del profesorado. Así, mientras los docentes de las áreas de lengua y ciencias sociales tienden a manifestar una concepción más incremental, procesual y multidimensional, los docentes del área de matemáticas se decantan por creer en una noción de inteligencia mucho más fija, unidimensional e innata.

En segundo lugar, y por lo que se refiere a la relación entre las concepciones de la inteligencia y la capacidad y las expectativas del profesorado en términos de género, origen social y etnicidad, hay consenso en señalar que dichas concepciones no son neutras en términos del origen social de los y las estudiantes. Tal como ha destacado de forma extensa la investigación sobre expectativas docentes (Diamond et al., 2004; Rist, 1970; Houtte, 2011), estas están cruzadas explícita o implícitamente por las variables de género, raza/ etnicidad y clase social. De este modo, la percepción escolar de la inteligencia y la capacidad no se puede entender al margen de las concepciones docentes sobre lo que es o lo que debería el «alumno ideal» y su mediación por variables de tipo social e institucional. Como señala Korp (2011: 35), la noción de inteligencia que tiene estatus hegemónico en la sociedad es típicamente valorada según criterios que reflejan los estilos cognitivos, lingüísticos y conductuales de las clases dominantes. Es por ello que, en términos de Oakes et al. (1997), la ideología de la inteligencia hace que formas particulares de capital cultural (o formas particulares de conocimiento) sean más reconocidas y valoradas dentro del ámbito escolar y, por ende, más fácilmente equiparables con las nociones dominantes de inteligencia y capacidad.

Es numerosa la investigación que demuestra que el alumnado cuyos conocimientos, formas de comunicación y modos de comportamiento dentro de la escuela se alejan de las pautas dominantes de las clases medias tiene más probabilidades de ser percibido como menos inteligente por parte de sus docentes (Nieto y Bode, 2007; Sue y Sue, 2007; Valenzuela, 1999). Es altamente significativo resaltar en este ámbito el estudio de Hatt (2007), ya que, si bien se centra en los discursos sobre la inteligencia que utilizan jóvenes de 18 a 24 en situación de elevada exclusión social y educativa en Estados Unidos, su análisis 
pone de manifiesto la existencia de dos grandes discursos sobre la inteligencia, la «inteligencia de libro» (book smart) y la «inteligencia de calle» (street smart), que cuestionan el discurso dominante sobre la inteligencia como inteligencia académica (o de libros) que opera en las escuelas. Tal como afirma la autora, «la forma a través de la cual ese construye la inteligencia en las escuelas es especialmente perjudicial para los y las jóvenes marginados en términos raciales, étnicos o económicos» (Hatt, 2007: 148). En definitiva, pues, la inteligencia que se valora y reconoce en la escuela tiende a excluir otras formas de conocimiento o capacidades que se alejan de los patrones culturales dominantes.

En tercer lugar, los resultados de las investigaciones desarrolladas en este campo ponen de manifiesto que las concepciones docentes sobre la inteligencia y la capacidad informan sus acciones, interacciones y prácticas pedagógicas y que, además, se institucionalizan en diferentes dispositivos/artefactos educativos. Las formas de agrupación del alumnado son, en particular, uno de los mecanismos clave a partir de los cuales se producen y reproducen las concepciones docentes sobre la habilidad de los y las estudiantes y, por ende, los que han recibido gran parte de la atención de la literatura especializada (Gamoran, 2009; Oakes et al., 1997).

Existe numerosa investigación, de hecho, que pone de manifiesto que las prácticas de agrupación del alumnado (ability grouping, en inglés) o de distribución de los mismos en diferentes itinerarios (tracking, en inglés) se basan directa e indirectamente en criterios de clasificación que pasan precisamente por identificar, clasificar y separar al alumnado según sus capacidades o habilidades. Estas capacidades se suponen claramente identificables y diferenciables, y para su detección se hace uso, de forma frecuente, de los resultados de los y las estudiantes en diversos exámenes y pruebas de competencia o nivel. Las notas, de hecho, utilizando la terminología de Korp (2011), funcionan como un símbolo de la inteligencia y la capacidad (signifier of smartness, en el original en inglés) que se tiende a asumir como puramente técnico, objetivo y neutral. A pesar de ello, es numerosa la investigación que señala que las formas de evaluar al alumnado son también producto de contextos culturales, sociales e institucionales específicos y que, por ende, están marcadas por relaciones de poder (Gillborn y Youdell, 2001). Es más, la numerosa investigación existente sobre las formas de agrupación y división del alumnado en grupos o itinerarios diferenciados pone de manifiesto el poderoso efecto que juegan las expectativas docentes no solo en las formas de detectar y clasificar las habilidades de diferentes tipos de alumnos, sino también en las prácticas curriculares, pedagógicas y de evaluación que se llevan a cabo en diferentes grupos e itinerarios (Castejón, 2017; Liou y Rotheram-Fuller, 2019). Es por ello —entre otros motivos-que los grupos de «bajo nivel» y los «itinerarios profesionales» de forma global están sobrerrepresentados por alumnos de bajo estatus socioeconómico y cultural, de origen migrado o de grupos étnicos minoritarios (Castejón, 2017; Boone y Houtte, 2013). Es también por ello que estos mismos grupos e itinerarios presentan modalidades pedagógicas mucho más centradas en el control del comportamiento y el aprendizaje de conceptos básicos que en la transmisión 
de conceptos teóricos de «orden superior» (Billett, 2016; Nylund y Rosvall, 2016). En el caso específico de la Formación Profesional, son diversas las investigaciones que señalan que, si bien la provisión curricular de este itinerario educativo se había caracterizado históricamente por diferentes formas de articulación entre teoría y práctica, se ha ido reduciendo progresivamente a aspectos puramente prácticos y procedimentales (Gamble, 2006; Nylund et al., 2017; Wheelahan, 2007).

En cuarto lugar, las investigaciones desarrolladas en este ámbito dedican una atención especial a analizar de qué manera las concepciones docentes sobre la capacidad de sus estudiantes y las prácticas pedagógicas que estas concepciones tienen asociadas impactan sobre la construcción de la identidad de los y las jóvenes. Los individuos siempre construimos nuestra identidad con relación a los otros y, por tanto, es siempre relacional. Las escuelas, en particular, tienen un rol central en la producción de las identidades de los y las jóvenes. Como señala Perry (2002, citado en Reay, 2010), las normas, prácticas y expectativas escolares proporcionan materiales simbólicos clave a partir de los cuales los y las estudiantes dan sentido a sus experiencias y se definen a sí mismos. En el campo específico que nos ocupa, la escuela es precisamente el lugar prioritario en el que los y las estudiantes se perciben y construyen a sí mismos como más o menos capaces o hábiles. De hecho, las concepciones docentes sobre la capacidad no solo informan, sino que básicamente posibilitan o limitan diferentes construcciones sobre la identidad de los y las jóvenes con claras consecuencias sobre su autoestima, sus disposiciones y sus decisiones educativas (Hatt, 2007). A modo de ejemplo, destaca el estudio etnográfico realizado por Valenzuela sobre los resultados académicos y las orientaciones escolares de jóvenes de origen mexicano en una escuela secundaria en Houston (Texas). Según muestran los resultados de su estudio, muchos de estos jóvenes no se sentían suficientemente «listos» para ir a la universidad precisamente porque durante toda su experiencia escolar habían sido tratados discursiva y materialmente como tales. Asimismo, el estudio de Nieto y Bode (2007) muestra que, a consecuencia del tracking, los y las estudiantes acaban creyendo que su pertenencia a los grupos de «menos nivel» es una consecuencia natural de su nivel de «inteligencia» $\mathrm{y}$ "capacidad», $\mathrm{y}$, por tanto, asumen que si están en dichos grupos es porque no son suficientemente listos. Es imprescindible, pues, preguntarse qué oportunidades tienen los y las estudiantes de construir su identidad académica en diferentes contextos escolares (Korp, 2011), ya que, como señala Youdell (2006), los procesos de exclusión escolar pasan por la construcción de identidades y subjetividades entre los y las jóvenes según las cuales se definen "cuerpos imposibles y yoes imposibles» (impossible bodies, impossible selves, de su original en inglés) y, por ende, futuros «imposibles».

\section{Metodología, muestra y procedimientos de análisis}

Como se ha señalado en la introducción, el objetivo del artículo es analizar la imagen que el profesorado tiene construida del alumnado de Bachillerato y 
de FP e investigar las características atribuidas a los y las estudiantes de ambos itinerarios formativos en términos de capacidades, hábitos y carácter.

Para llevar a cabo el análisis, se han realizado 37 entrevistas semiestructuradas con profesorado de 8 centros educativos de Barcelona. Concretamente, la muestra está conformada por tutores y tutoras de $4 .^{\circ}$ de ESO y $1 .^{\circ}$ de Bachillerato y FP. ${ }^{4}$ Los centros educativos han sido seleccionados porque disponen, dentro de su oferta educativa, de la etapa de Educación Secundaria Obligatoria además de los dos itinerarios formativos de la etapa posobligatoria. Si bien los centros que cumplen con esta característica no son lo más habitual en Barcelona (el mapa educativo de la ciudad está configurado por centros con una oferta más bien segmentada, donde los que ofrecen ESO y Bachillerato son mayoría, y otros disponen solamente de FP), se han seleccionado escuelas con las dos vías formativas posobligatorias para realizar un análisis relacional de ambos itinerarios. En la selección se ha tenido también en cuenta la diversidad de los centros educativos, en términos de localización y composición social. De los 8 centros seleccionados, uno es de titularidad privada y los demás son públicos. El análisis forma parte de un proyecto de investigación más amplio, orientado a comprender los factores que explican las transiciones a la educación secundaria posobligatoria en contextos urbanos, ${ }^{5}$ y que incluye entrevistas con diferentes actores educativos, cuestionarios con jóvenes y observaciones de jornadas de puertas abiertas. ${ }^{6}$

Las entrevistas con los y las tutoras, de una duración aproximada de 60 minutos, se han llevado a cabo durante el curso 2018-2019 en los propios institutos y se han registrado en formato audio para su posterior transcripción. Las entrevistas con los y las tutoras constan de diferentes bloques temáticos, entre los que se incluyen aspectos vinculados a la trayectoria profesional de los/as entrevistados/as, las características del centro educativo en el que trabajan y del grupo clase del que son responsables, las opiniones sobre el estado de la educación posobligatoria, el perfil de los y las estudiantes de diferentes itinerarios educativos o los motivos que explican las elecciones y transiciones educativas de los y las jóvenes, entre otros. Para la realización de este artículo, el análisis de las entrevistas se ha centrado en los discursos docentes sobre los y las estudiantes de los diferentes itinerarios formativos y, en particular, se ha llevado a cabo una codificación basada en res grandes dimensiones de análisis: capacidades, hábitos y carácter. Siguiendo la aproximación de la grounded theory, la categoría de la capacidad se ha construido deductivamente, mientras que las de los hábitos y el carácter han emergido de forma inductiva a partir del análisis de las entrevistas. Para cada concepto se han abordado cuatro gran-

4. Concretamente, se han realizado 14 entrevistas a tutores/as de $4 .^{\circ}$ de ESO y $1 .^{\circ}$ de Bachillerato y 22 entrevistas a tutores/as de FP.

5. Proyecto EDUPOST16, cuya referencia completa puede encontrarse en la introducción del artículo.

6. Concretamente, se han realizado 28 entrevistas con policy makers, 35 con equipos directivos y de coordinación de los institutos seleccionados, 37 con tutores y tutoras, y 68 con jóvenes, 25 observaciones en jornadas de puertas abiertas y 1.319 cuestionarios con jóvenes de $1 .{ }^{\circ}$ de Bachillerato y FP. 
des dimensiones: las definiciones hechas por el profesorado; la identificación discursiva de diferentes tipologías; la relación de estos conceptos con el perfil social de los y las estudiantes, y su relación con el itinerario educativo que están cursando los y las jóvenes.

\section{Resultados: concepciones docentes sobre el alumnado}

En este apartado, se presentan los resultados del análisis con el objetivo de comprender las concepciones y atribuciones docentes sobre los y las estudiantes de los diferentes itinerarios formativos posobligatorios: Bachillerato y FP. El análisis se estructura en tres grandes apartados: en primer lugar, se presenta la concepción global que los docentes tienen de la inteligencia/capacidad; en segundo lugar, se identifican dos grandes perfiles de alumnado, construidos según dos grandes concepciones de las capacidades (las académicas y las prácticas); en tercer lugar, se introducen los discursos docentes en relación con los hábitos y el carácter de los y las estudiantes.

\subsection{La capacidad como algo natural y objetivable}

El primer aspecto que se deriva del análisis es una concepción docente altamente naturalizadora de las capacidades y las habilidades de los y las estudiantes. Esta concepción, expresada fundamentalmente a través de teorías implícitas, asume que la capacidad es algo que viene mayormente dado y que no depende de la voluntad de docentes ni estudiantes. De este modo, entre los discursos docentes predomina la idea de que los alumnos «son», «valen» o «sirven» para diferentes ámbitos, como si fuera una cuestión innata e inamovible. Se realiza, así, una especie de asunción de que los y las estudiantes deben aceptar aquello para lo que valen y aquello para lo que no, tal y como se pone de relieve en las siguientes citas.

Del mismo modo que se acepta la realidad de que si yo quiero ser bailarina y no tengo dones no voy a bailar, o voy a bailar muy mal, y no pasa nada, pues no me dedicaré al baile y nadie se rasga las vestiduras. Pues del mismo modo hay alumnado que no tiene capacidades. Y de estos tenemos aquí. Es muy difícil aceptar... (C6, tutora de FP) ${ }^{7}$

Hay un tema innegable, que es el tema de las capacidades, y allí está... Y nos negamos a veces a afrontarlo, porque, claro, a nadie le gusta que le digan que su hijo tiene dificultades, y, claro, aquí eufemismos los que quieras. Pero es que al final, es la vida. (C2, tutora de Bachillerato)

Tal como se pone de manifiesto en la primera cita, las «capacidades» son interpretadas desde una perspectiva de dotes de las que los individuos disponen

7. Los nombres de los centros se han sustituido por una clasificación numérica para garantizar el anonimato. 
o carecen, como si de un designio de la naturaleza se tratara. Esta idea está estrechamente vinculada a la de los dones naturales a los que hacen referencia Bourdieu y Passeron (2009 [1964]) cuando argumentan que, a menudo, los resultados educativos de los y las estudiantes son interpretados en términos de un orden supuestamente natural que omite los efectos de origen social y en particular de la estructura de capitales (económico, social, cultural y simbólico). De este modo, expresiones como «no se le da bien» o "no sirve para esto» ponen de manifiesto que el profesorado tiende a dar por supuesto que las capacidades son concedidas por la naturaleza, como si no fueran producto también de la acción educativa y como si no pudieran ser modificadas.

Es más, cuando los docentes establecen una relación discursiva entre las capacidades de los y las estudiantes y sus contextos socioeconómicos y culturales de referencia, suelen hacerlo de forma ampliamente estática y fija, de manera que reproducen así las teorías del déficit que presuponen que la inteligencia se explica de forma casi mecánica por los modos de socialización familiar. De este modo, se tiende a normalizar las ventajas educativas que otorgan determinadas posiciones sociales, sin problematizar el efecto de las desigualdades sociales ni cuestionar el papel que la institución escolar puede tener en la reproducción de las mismas.

Los alumnos que han sido escolarizados aquí, todos han tenido las mismas oportunidades. O sea, no existe ningún tipo de diferencia, depende de lo que ellos quieran hacer, si quieren estudiar más o menos [...]. A veces depende de la situación familiar, pero en general yo veo que normalmente tienen las mismas oportunidades. ( $\mathrm{C} 3$, tutora de $4 .^{\circ}$ de $\mathrm{ESO}$ )

Esta misma lógica también opera en el caso del género, especialmente cuando se hace alusión a la mayor proporción de chicos o chicas en determinados itinerarios formativos (por ejemplo, mayor proporción de chicas en la FP de ámbito sociosanitario o mayor proporción de chicos en el ámbito tecnológico). Se trata de una forma eminentemente acrítica y descontextualizada de hacer referencia a las desigualdades de género. Tal y como se ilustra en la cita, se alude a aspectos como la tradición o la costumbre para justificar estas diferencias en las elecciones de los y las jóvenes, que se corresponden con la división sexual del trabajo.

Los chicos tiran hacia la rama tecnológica, muchos. Mientras que las chicas tienen más tendencia hacia la parte de humanidades y sociales. Lo que no quiere decir que, como siempre, haya excepciones. Pero los chicos prefieren más informática, ingenierías... Supongo que por tradición siempre han sido estudios masculinos. (C3, tutora de $4 .^{\circ}$ de ESO)

Finalmente, es importante señalar la relación discursiva que se establece entre las capacidades del alumnado, los resultados de las evaluaciones y la orientación hacia una u otra vía formativa. El análisis de las entrevistas pone de manifiesto que, de forma global, las notas se perciben como una forma 
objetiva y neutral de medir las capacidades que, a su vez, sirven de proxy para orientar al alumnado hacia Bachillerato o FP. Así, los y las jóvenes que no tienen buenos resultados académicos durante la ESO son orientados de forma sistemática hacia la FP, mientras que el alumnado que tiene buenas notas es mayoritariamente orientado hacia Bachillerato, ya que se supone que tiene las capacidades para seguir esta vía formativa con éxito. Es, pues, altamente excepcional que se proponga a un «buen alumno» escoger la vía profesional.

Claro, un alumno que tiene resultados no demasiado buenos académicamente y pretende hacer según qué tipo de estudios, un Bachillerato por ejemplo, pues claro, se le asesora y se le dice: «Mira, ahora mismo no es lo que más te conviene, busca algo que se adapte mejor a tus capacidades». (C3, tutora de 4. ${ }^{\circ}$ de $\left.\mathrm{ESO}\right)$

Tuvimos una promoción, no hace mucho, de un grupo muy importante de chicos muy capacitados y con muy buenas notas que decidieron cursar un ciclo [FP]. Y no se lo recomendamos, ¡eh! (C6, tutora de Bachillerato)

\subsection{El alumnado «académico» y el alumnado "práctico»}

En la línea de lo que se ha comentado, las capacidades son consideradas intrínsecas a cada individuo, ya sea por una cuestión innata o por una naturalización de las ventajas derivadas de la posición social. De acuerdo con esta concepción, el profesorado tiende a construir dos perfiles de jóvenes claramente dicotómicos: el alumnado "académico», al que se le atribuye una mayor capacidad/ habilidad para producir pensamiento elaborado o para adquirir conocimientos abstractos, y el alumnado "práctico», al que se considera más apto para el trabajo manual. De hecho, esta dicotomía, dada la concepción esencialista y naturalizadora de las capacidades, se considera como algo natural, dada por obvia, y escasas veces es cuestionada por parte del profesorado.

Yo no tengo ni idea de cómo se desarrolla la inteligencia [...]. Pero, hombre, si todos fuéramos igual de listos o de tontos, dicho así simplificado y de una forma muy vulgar, pues... Somos diferentes, ¿no? Lo que sí veo son capacidades diferentes. $\mathrm{O}$ sea, gente que no tiene herramientas de estudio y en cambio será un buen profesional. (C6, tutora de FP)

Es más, como se pone de manifiesto en la cita anterior, la diferencia entre capacidades teóricas y prácticas se percibe como una cuestión puramente descriptiva que no está mediada por los efectos de la desigualdad social. Así, según una lógica ampliamente funcionalista, se supone que cada persona dispone de diferentes capacidades teóricas o prácticas que además son útiles y necesarias para ejercer las diferentes funciones que requiere la división social del trabajo en las sociedades contemporáneas. De este modo, se omite que las capacidades académicas y prácticas no gozan del mismo reconocimiento ni del mismo prestigio en la estructura escolar ni social. Un prestigio que se explica por la 
jerarquía existente entre el conocimiento abstracto-académico y el conocimiento práctico (Bernstein, 2000; Young, 2006), y que, a su vez, responde a las atribuciones que diferentes agentes educativos y sociales hacen a cada tipo de conocimiento y a los vínculos entre los tipos de conocimiento y un mercado de trabajo altamente segmentado y estratificado (Tarabini y Jacovkis, 2019).

Las entrevistas realizadas con los y las tutoras de ESO, Bachillerato y FP también han puesto de manifiesto que esta dicotomía condiciona ampliamente qué itinerario (FP o Bachillerato) encaja con cada perfil de alumnado: el alumnado "académico» sería el que encajaría con el Bachillerato, mientras que al alumnado "práctico» le correspondería cursar FP. Esta idea se sostiene en una creencia ampliamente generalizada entre el profesorado de que existe también una dicotomía entre el tipo de conocimiento que predomina en cada vía formativa: el conocimiento teórico, abstracto, conceptual que define el Bachillerato, y el conocimiento práctico y aplicado que caracteriza la FP. Como hemos argumentado en otra ocasión (Tarabini y Jacovkis, 2019), el primer tipo de conocimiento, el teórico, se considera difícil, complejo y menos accesible, y estaría pensado para el alumnado "académico»; mientras que el segundo, considerado más fácil y accesible, sería adecuado para el alumnado "práctico». Así pues, como se refleja en las siguientes citas, cada vía formativa sería adecuada para un tipo de alumnado particular en función del encaje entre «tipos de conocimiento» $\mathrm{y}$ «tipos de inteligencia/capacidad».

Tú ya ves si un alumno es académico o no. Al final, el Bachillerato es muy académico, si tienes alumnos que ya han tenido dificultades de poder rendir... [...]. Claro, no quiere decir que los ciclos [FP] no sean académicos, que lo son, pero hay un porcentaje, muy alto, manipulativo, mucho más vivencial... (C2, tutora de Bachillerato)

En el Bachillerato las materias son muy abstractas, y ellos a lo mejor estarían mejor estudiando un ciclo $[\mathrm{FP}]$ sobre informática, sobre temas más prácticos. (C4, tutor de Bachillerato)

[Los ciclos de FP] son más manipulativos, más de prácticas. Van a las empresas, trabajan... Son más atractivos para una persona a la que lo académico no le gusta. (C6, tutora de Bachillerato)

De este modo, lo que explicaría la elección de Bachillerato o FP serían principalmente las capacidades de las que dispone el alumnado para acceder a un tipo de conocimiento u otro, y se omitiría así el efecto que juegan los factores sociales, institucionales o culturales en la explicación de las elecciones posobligatorias de los y las jóvenes (Tarabini e Ingram, 2018).

Del discurso docente se desprende también la idea de que el conocimiento teórico, el de Bachillerato, no es accesible para todo el mundo y, por tanto, la orientación hacia FP se hace a menudo desde una lógica de descarte. Es decir, se considera la mejor opción para aquellos que «no son buenos» con el conocimiento abstracto. Esto explicaría que, en los discursos docentes, el alumnado de FP, 
el «práctico», se construya habitualmente en oposición al alumnado de Bachillerato, el «académico». En este sentido, mientras que al alumnado "académico» se le define con un conjunto de atributos positivos acerca de sus «capacidades intelectuales», las características definitorias del alumnado "práctico» serían sobre todo no estar suficientemente dotado, eso es, no tener las "capacidades intelectuales» necesarias para emprender estudios de Bachillerato.

Se orienta a ciclos $[\mathrm{FP}]$ a quien no puede o quien no tiene pronóstico de éxito en el Bachillerato. Entonces, normalmente, a quien en la ESO tiene una buena media, va tirando o destaca, se le recomienda Bachillerato. (C2, tutora de FP)

Hay un dicho popular que dice: «El que vale, vale, y el que no, a FP». Solo con este dicho ya te estoy diciendo lo que la sociedad te dice: si puedes estudiar, si se te da bien, haz Bachillerato, que no puedes... Pues FP. Y esto no debería ser así... (C7, tutor de FP)

Cuando yo era tutor de la ESO, los compañeros te decían: este alumno no sirve para estudiar, pues venga, a un ciclo. (C5, tutor de FP)

Así lo explica esta tutora que, si bien reproduce la idea de los dones naturales, reconoce también una falta de reconocimiento de las aptitudes del alumnado «más manipulativo».

Como profesores y como padres, dices: «Mira, este chico, como no tira, vamos a intentar encararle hacia ciclos [FP]» [...]. Yo creo que, en vez de decir «no tira», tendríamos que decir: «Mira, como esta persona es manipulativa, tiene un perfil más...» [...]. Tendríamos que buscar palabras para definir a los alumnos en positivo cuando tengan una aptitud buena para la FP. Claro, si tienes una aptitud buena para la FP no tiene que ser con desprestigio de otra: «Mira, este chico tiene una habilidad manual, y una precisión, y una paciencia que para hacer un ciclo [FP] sería espléndido». No. Decimos: «Como no tira, pongámoslo en un ciclo [FP]». Entonces, claro, estamos favoreciendo algo que no tiene en vez de algo que tiene. (C2, tutora de Bachillerato)

\subsection{Más allá de las capacidades: formas de estar y ser en las aulas}

Como se ha mostrado hasta ahora, las capacidades, concebidas como dones naturales o la naturalización de las desigualdades, son un elemento que diferencia claramente al alumnado "académico" y al alumnado "práctico", al de Bachillerato y al de FP. Esta dicotomía, sin embargo, no se ciñe exclusivamente a las capacidades/habilidades, digamos cognitivas, sino que existen más diferencias entre el tipo de rasgos atribuidos a cada uno de estos perfiles. Los «hábitos de estudio» y el «esfuerzo» son, en particular, dos cualidades centrales que, según los docentes, caracterizan a los y las jóvenes «académicos», y de las que, a menudo, carecen los y las alumnas que no pertenecen a esta categoría. Así, y según se desprende del análisis de los discursos docentes, para cursar Bachillerato con éxito no solo hace falta ser un estudiante «académico», sino 
que además es imprescindible tener «hábitos» y «esforzarse». Dicho de otro modo, si eres «listo» pero no te esfuerzas, el Bachillerato no es tu lugar. Por el contrario, se sobreentiende que aquellos alumnos con falta de hábitos de estudio, mal comportamiento o falta de esfuerzo solo pueden cursar FP.

Hay alumnos que van con una inercia, que hacen toda la ESO aprobando por los pelos [...]. A estos se les recomienda que hagan un ciclo formativo [FP], pero no por nivel, sino porque no han querido trabajar nunca, y el Bachillerato les costará. (C3, tutor de Bachillerato)

Es necesario tener unas características determinadas para poder estudiar un Bachillerato y después una carrera y todo [...]. Tienes que tener la capacidad de adaptarte al mundo académico, a los libros, a tener el culo pegado en una silla muchas horas, de escuchar con atención sin distraerte [...]. Entonces, a la gente que no tiene esta capacidad se la intenta orientar hacia otro lado. (C4, tutor de Bachillerato)

En este sentido, los docentes de FP lamentan que entre el alumnado de la vía profesional exista una elevada proporción de jóvenes que acumulan trayectorias de suspensos, de repeticiones y que llegan con bajos niveles de competencias académicas. Así, lamentan que, cuando empiezan la FP, se encuentran con un perfil de estudiantes con fuertes dificultades de aprendizaje como consecuencia de lo que interpretan como un débil nivel de exigencia en las etapas educativas anteriores, tal como argumentan los docentes en las siguientes citas.

Te llegan algunos alumnos con la ESO aprobada y dices: «Pero ¿tú cómo has llegado hasta aquí?». Porque es que no... No, no. Son cosas muy básicas y no. Aspectos de lógica y matemática, y dices: «Es que no... Y ¿̇cómo puede ser que tú hayas aprobado la ESO?». Entonces, claro, se ponen a hacer un ciclo [FP], y yo creo que tienen la misma sensación que en la $\mathrm{ESO}$, que les aprobarán algo así porque sí. (C6, tutor de FP)

Yo creo que hay muchos que llegan aquí y los profesores dicen: «Qué hago, qué hago, qué hago». Los de antes no han hecho nada (profesorado de etapa primaria - secundaria obligatoria), y es como una cadena de montaje, y van pasando. Y entonces me llegan a mí y te dicen: «No, no, si se trata de que hagan algo». (C5, tutor de FP)

Los ves como hacen las cosas y dices: «No me puedo creer que no hayas repetido ningún curso. No me puedo creer que tú hayas llegado aquí por la ESO». Y los ves escribir y dices: «Es que no tienes ni idea, es que yo no creo que esta persona pueda tratar ya no en el medio natural, yo a ti no te dejo a mi hijo ni harto de vino». (C5, tutor de FP)

Se pone de manifiesto, pues, la disyuntiva de que el alumnado de FP no puede optar por la vía académica por su trayectoria y su perfil educativo, pero ello conlleva que la FP se convierta en un espacio con una mayor concentración 
de complejidades sociales, lo que, a su vez, implica un mayor desprestigio de esta vía formativa.

En conjunto, el análisis pone de manifiesto que, de forma global, el profesorado tiende a considerar que el alumnado de Bachillerato presenta unas disposiciones, unos hábitos de trabajo y una forma de comportarse en el aula más apropiados para el aprendizaje, y que, por tanto, es este tipo de alumno el que marca el patrón de normalidad al que el resto de los y las estudiantes se debe ajustar. Estas disposiciones, sin embargo, no están al margen del origen social y muestran una mayor alineación con el alumnado perteneciente a las clases medias (Auwarter y Aruguete, 2008; Dunne y Gazeley, 2008), hecho que explicaría que estos jóvenes sean orientados de forma sistemática hacia el Bachillerato por parte del profesorado.

Otro elemento que se desprende del análisis de las entrevistas está vinculado a la interpretación que realizan los docentes sobre el «interés» y la "motivación» de los y las jóvenes con respecto a sus estudios. En este aspecto, se observan diferencias sustanciales según el itinerario formativo al que se hace referencia. Así, se pone mucho más en cuestión si los y las estudiantes de FP están o no motivados; en cambio, en el caso de los y las estudiantes de Bachilleratos se da por supuesto que están interesados. En general, según el profesorado, los y las estudiantes de FP son más indecisos, tienen menos claro qué quieren hacer, están más desmotivados, están «más por estar», están aquí como podrían estar haciendo cualquier otra cosa o eligen un ciclo de FP porque les queda cerca de su casa.

Antes la gente que estudiaba administrativo lo estudiaba porque le gustaba. Ahora, preguntas, y la mayoría no saben por qué estudian eso. Claro, esto ya implica una desmotivación, porque, claro, tu les preguntas por qué están aquí y algunos te dicen que han cogido esto como cualquier otra cosa [...]. Que podrían hacer perfectamente peluquería. (C7, tutor de FP)

En cambio, en los discursos del profesorado, no se cuestiona si hay estudiantes de Bachillerato que estén también "por estar» o que no tengan claro que es lo que quieren hacer con su vida. Asimismo, cuando se discute sobre los posibles factores que explican el mayor éxito o fracaso escolar de cada vía formativa (promoción, graduación, notas), se hace una mención explícita al interés como condición primordial para tener «éxito» en la FP, pero en cambio parece que para el caso del Bachillerato es menos relevante tener interés para poder sostener esta vía educativa con éxito.

El interés y la motivación, pues, son interpretados desde una perspectiva de juicio moral, de forma tal que está altamente penalizado no estar interesado o motivado por los estudios. Si bien las capacidades/habilidades se asume que se tienen o no se tienen, en el caso del interés y la motivación se observa una fuerte desaprobación hacia los y las estudiantes que no muestran interés por aquello que están estudiando. De algún modo se asume, pues, que, si bien la capacidad no se puede controlar, el interés depende exclusivamente de la voluntad individual. 
Un último elemento que se desprende del análisis es la alusión a cuestiones de carácter para explicar la elección de determinados itinerarios formativos. En este sentido, en múltiples ocasiones se interpreta que un estudiante «ha nacido» para estudiar una determinada formación. Del mismo modo que se mencionaba anteriormente para el caso de las capacidades/habilidades, el carácter se explica como un designio de la naturaleza. El recurso del carácter para describir al alumnado es especialmente significativo en algunas modalidades formativas, entre las que destacan el Bachillerato artístico y la FP del ámbito sociosanitario. En estos casos, de hecho, parecería que tener un «carácter especial» es un requisito imprescindible para realizar con éxito dichas modalidades formativas. Tal como se pone de relieve en las siguientes citas, el profesorado considera que para estudiar determinadas ramas es necesario contar con unos rasgos de personalidad determinados, como por ejemplo la empatía, la creatividad, la amabilidad o la responsabilidad.

Auxiliar de enfermería es un trabajo totalmente vocacional, entonces son personas que deben tener ciertas características, llamémoslas psicológicas, un carácter especial. No todo el mundo es capaz; sobre todo les pasa cuando empiezan a hacer las prácticas, que se dan cuenta de que aquello es o no es lo suyo. (C1, tutora de FP)

Esta parte más, más artística les viene mucho por un tema de sensibilidad. Suelen ser gente con tacto, con mucha habilidad manual, con una fuerte sensibilidad, con mucho interés por lo creativo, por la fotografía, por el vídeo... Suelen tener una curiosidad muy grande. (C1, tutor de Bachillerato)

En este sentido, es importante mencionar que cuando se indaga sobre los motivos que pueden explicar esta construcción del carácter o la personalidad, si bien aparecen cuestiones de tipo social o cultural, estas se describen desde una perspectiva también naturalizadora. A modo de ejemplo, cuando se menciona la mayor presencia de mujeres en la FP del ámbito sociosanitario, se da por descontado que las mujeres tienen una mayor vocación hacia el cuidado de las personas, pero no se cuestionan las consecuencias ni se problematizan las causas de tales diferencias entre hombres y mujeres. De forma similar, en el caso de los y las estudiantes que cursan el Bachillerato artístico, si bien la variable capital cultural no se hace explícita, se hace mención a un proceso de "cultivación" por parte de los progenitores como uno de los principales factores que explican la disposición de un carácter creativo, curioso y sensible.

Cuando me reúno con los padres, sí que es verdad que muchos te dicen pues que al niño le había gustado siempre dibujar y «una vez fuimos a una feria del cómic y una vez fuimos a un museo y había dibujos, y una vez cuando nos fuimos de vacaciones vimos una exposición de...». O sea, que los padres, este lado más artístico, tal vez inconscientemente hace muchos años, lo han ido cuidando, lo han ido regando poco a poco. (C1, tutor de Bachillerato) 
En definitiva, en esta naturalización de capacidades, hábitos y carácter de los y las estudiantes se tiende a omitir de qué forma las trayectorias educativas, el paso por el instituto, la relación con el profesorado, factores vinculados al origen social, el género o la etnia o la propia estructura de la educación secundaria posobligatoria pueden estar contribuyendo a explicar los diferentes itinerarios formativos en los que están escolarizados diferentes perfiles de estudiantes.

\section{Conclusiones}

El análisis realizado en este artículo partía del interés de estudiar el rol del profesorado en la configuración de las pautas de desigualdad social asociadas con las transiciones de los y las jóvenes a la educación secundaria posobligatoria. Sin duda, los factores que explican la relación entre itinerarios educativos y desigualdad social son múltiples y diversos y se asocian con elementos de índole institucional, económica, social y cultural. Las creencias docentes, en particular, juegan un rol crucial para entender la asignación de diferentes estudiantes a diferentes itinerarios. Como ha señalado la literatura internacional, las expectativas docentes tienen impactos en la autoestima, la actitud y los resultados de los y las estudiantes, y, en términos globales, contribuyen a explicar por qué los itinerarios profesionales tienden a estar sobrerrepresentados por chicos de bajo estatus socioeconómico y cultural, de origen migrado o de grupos étnicos minoritarios.

El objetivo específico del artículo, enmarcado en una perspectiva constructivista, ha sido analizar la imagen que el profesorado tiene construida del alumnado de Bachillerato y de FP, y destacar concretamente las características atribuidas a los y las estudiantes de ambos itinerarios formativos en términos de capacidades/habilidades, hábitos y carácter. De los resultados del análisis se desprenden tres grandes conclusiones.

En primer lugar, los y las docentes entrevistados tienden a movilizar teorías implícitas sobre la inteligencia y la capacidad que las conciben como algo mayoritariamente natural, derivado de las características intrínsecas de los y las estudiantes y de sus contextos de referencia. Ello supone que la inteligencia o las capacidades se consideren como "propiedades esenciales", de las que los individuos disponen o carecen, fruto de la naturaleza o del azar, y se obvie el efecto de factores contextuales, sociales o institucionales en el desarrollo de las mismas. Es más, como se ha demostrado, cuando los docentes establecen una relación discursiva entre las capacidades de los y las estudiantes y sus contextos socioeconómicos y culturales de referencia suelen hacerlo de forma ampliamente estática y fija, de modo que reproducen las teorías del déficit que atribuyen a los modelos de socialización familiar, de forma casi mecánica, las capacidades de los individuos. Asimismo, se ha puesto de manifiesto el rol que juegan las notas como artefacto a través del cual evidenciar la existencia de diferentes capacidades y legitimar la orientación de los y las estudiantes hacia diferentes vías formativas. De 
este modo, los y las estudiantes que tienen buenas notas durante la ESO son mecánicamente representados como "buenos estudiantes» y, por ende, derivados al Bachillerato. Por el contrario, el alumnado que acumula suspensos, repeticiones y malas notas durante la ESO es considerado como «incapaz» de realizar la vía académica y, por tanto, es sistemáticamente orientado hacia la FP.

En segundo lugar, del análisis de los discursos docentes se identifica una construcción ampliamente dicotómica entre dos tipos de capacidades/ habilidades de los y las estudiantes, las «teóricas» y las «prácticas», que, a su vez, se corresponden con el tipo de conocimiento, «abstracto» o "aplicado», que se supone que caracteriza a cada itinerario educativo. Del análisis de las entrevistas, de hecho, se desprende una asociación discursiva ampliamente lineal entre "tipos de alumnos» $\mathrm{y}$ "tipos de itinerarios», de acuerdo con un supuesto encaje natural entre «tipos de conocimiento" y «tipos de capacidad/ habilidad». No se trata solo, como decíamos antes, de que los alumnos «más capacitados», los que tienen mejores notas, se consideren los más adecuados para la vía académica, sino que — casi de forma natural - se supone que los «alumnos teóricos» pertenecen al mundo del Bachillerato y «los alumnos prácticos» al mundo de la FP. Asimismo, el análisis pone de manifiesto una construcción discursiva de las capacidades teóricas y prácticas como si fueran puras diferencias que no se asocian con las con pautas de desigualdad social. De este modo, a partir de un discurso del «respeto a la diferencia» se acaba obviando y legitimando que el conocimiento teórico y el práctico no gozan del mismo prestigio dentro del orden social y escolar y, por ende, se acaba reproduciendo la desigualdad.

En tercer lugar, el análisis pone de manifiesto que la asociación discursiva entre «tipos de alumnos» $y$ «tipos de itinerario» no se justifica solo según las capacidades/habilidades, sino también en función de rasgos de conducta y carácter. Es decir, para ser alumno de Bachillerato o de FP no solo hace falta ser más o menos listo, tener unas u otras capacidades, sino también tener unos hábitos específicos, mostrar determinadas disposiciones escolares y disponer — principalmente en algunas especialidades - de determinados rasgos de personalidad que te habiliten para la adquisición de determinadas competencias y el posterior ejercicio de la práctica profesional.

Estos tres elementos, en conjunto, juegan un rol clave en la legitimación discursiva de un sistema de educación posobligatoria altamente segmentado y desigual, tanto en términos de funciones, contenidos y procesos pedagógicos como de provisión de la oferta y de sistemas de acreditación (Tarabini y Jacovkis, 2019). Como se ha argumentado, las creencias y las atribuciones docentes tienen un impacto central en la configuración de diferentes oportunidades de transición a la educación posobligatoria y, por tanto, ponen de relieve la centralidad de desplegar líneas de política educativa que habiliten para una práctica pedagógica reflexiva en el día a día de los centros de enseñanza. 


\section{Referencias bibliográficas}

Agirdag, Orhan; Houtte, Mieke van; Avermaet, Piet van (2012). «Ethnic School Segregation and Self-Esteem: The Role of Teacher-Pupil Relationships». Urban Education, 47 (6), 1135-1159.

$<$ https://doi.org/10.1177/0042085912452154>.

AnYon, Jean (1981). «Social Class and School Knowledge». Curriculum Inquiry, 11 (1), 3-42. <https://doi.org/10.1080/03626784.1981.11075236>.

Auwarter, Amy E.; Aruguete, Mara S. (2008). "Effects of Student Gender and Socioeconomic Status on Teacher Perceptions». The Journal of Educational Research, 101 (4), 243-246. <https://doi.org/10.3200/JOER.101.4.243-246>.

Bergh, Linda van den; Denessen, Eddie; Hornstra, Lisette; Voeten, Marinus; Holland, Rob W. (2010). «The Implicit Prejudiced Attitudes of Teachers: Relations to Teacher Expectations and the Ethnic Achievement Gap». American Educational Research Journal, 47 (2), 497-527. $<$ https://doi.org/10.3102/0002831209353594>.

Bernardi, Fabrizio; Requena, Miguel (2010). "Desigualdad y puntos de inflexión educativos: el caso de la educación post-obligatoria en España». Revista de Educación, número extraordinario, 93-118.

Bernstein, Basil (2000). Pedagogy, Symbolic Control and Identity: Theory, research, critique. Lanham (MD): Rowman \& Littlefield.

Billett, Stephen (2016). «Beyond competence: an essay on a process approach to organising and enacting vocational education». International Journal of Training Research, 14 (3), 197-214. <https://doi.org/10.1080/14480220.2016.1254365>.

Blossfeld, Hans-Peter; Shavit, Yossi (2011). «Persisting Barriers: Changes in Educational Opportunities in Thirteen Countries». En: Arum, Richard; Beattie, Irenee R.; Ford, Karly (eds.). The structure of Schooling. Readings in Sociology of Education. Londres: SAGE.

Bonizzoni, Paola; Romito, Marco; Cavallo, Cristina (2016). «Teachers' Guidance, Family Participation and Track Choice: The Educational Disadvantage of Immigrant Students in Italy». British Journal of Sociology of Education, 37 (5), 702-720. <https://doi.org/10.1080/01425692.2014.982860>.

Boone, Simon; HoutTe, Mieke van (2013). "Why are teacher recommendations at the transition from primary to secondary education socially biased? A mixed-methods research". British Journal of Sociology of Education, 34 (1), 20-38. <https://doi.org/10.1080/01425692.2012.704720>.

Bourdieu, Pierre; Passeron, Jean-Claude (2009 [1964]). Los Herederos: los estudiantes y la cultura. Madrid: Siglo Veintiuno Editores.

CASTEjón, Alba (2017). Expectativas docentes, agrupamiento del alumnado y segregación escolar. Una etnografía en entornos de alta complejidad social en Cataluña. Tesis doctoral. Universitat Autònoma de Barcelona.

Connell, Raewyn (1997). Escuelas y justicia social. Madrid: Morata.

Demanet, Jannick; Houtte, Mieke van (2012). «Teachers' attitudes and students' opposition. School misconduct as a reaction to teachers' diminished effort and affect». Teaching and Teacher Education, 28, 860-869. <https://doi.org/10.1016/j.tate.2012.03.008>.

Diamond, John B.; Randolph, Antonia; Spillane, James P. (2004). «Teachers' Expectations and Sense of Responsibility for Student Learning: The Importance 
of Race, Class, and Organizational Habitus». Anthropology \& Education Quarterly, 35 (1), 75-98.

<https://doi.org/10.1525/aeq.2004.35.1.75>.

Dunne, Mairead; Gazeley, Louise (2008). «Teachers, social class and underachievement». British Journal of Sociology of Education, 29 (5), 451-463. <https://doi. org/10.1080/01425690802263627>.

Gamble, Jeanne (2006). «Theory and Practice in the Vocational Curriculum». En: Young, Michael; Gamble, Jeanne. Knowledge, curriculum and qualifications for South African Further Education. Ciudad del Cabo: HSRC Press.

Gamoran, Adam (2009). Tracking and Inequality: New Directions for Research and Practice. WCER Working Paper, No. 2009-6. Madison: University of WisconsinMadison, Wisconsin Center for Education Research.

García, Enrique; Lorente, Rocío (2015). «Recorrido por la imagen social de la formación profesional: un camino hacia su revalorización». Revista Española de Educación Comparada, 26, 119-134.

DOI: $<10.5944 /$ reec.26.2015.14270>.

GARDNER, Howard (1988). «Beyond IQ: Education and human development». Harvard Educational Review, 57, 187-193. <https://doi.org/10.17763/haer.57.2.1210118834750615>.

Gillborn, David; Youdell, Deborah (2001). «The New IQism: Intelligence, 'Ability' and the Rationing of Education». En: Demaine, Jack (ed.). Sociology of Education Today. Londres: Palgrave Macmillan UK.

Hamilton, Lorna (2006). "Implicit theories of ability: Teacher constructs and classroom consequences». Scottish Educational Review, 38 (2), 200-212.

HATт, Beth (2007). «Street smarts vs. book smarts: the figured world of smartness in the lives of marginalized, urban youth». The Urban Review, 39 (2), 145-166. <https://doi.org/10.1007/s11256-007-0047-9>.

- (2012). "Smartness as a Cultural Practice in Schools». American Educational Research Journal, 49 (3), 438-460. <https://doi.org/10.3102/0002831211415661>.

HoutTe, Mieke van (2011). "So where's the teacher in school effects research? The impact of teachers' beliefs, culture, and behavior on equity and excellence in education». En: Branden, Kris van den; Avermaet, Piet van; Houtte, Mieke van (eds.). Equity and excellence in education: Towards maximal learning opportunities for all students. Nueva York (NY): Routledge.

Houtte, Mieke van; Demanet, Jannick; Stevens, Peter A. J. (2013). «Curriculum tracking and teacher evaluations of individual students: selection, adjustment or labeling?». Social Psychology of Education, 16 (3), 329-352. <https://doi.org/10.1007/s11218-013-9216-8>.

INE (Instituto Nacional de Estadística) (2019). INE Base, Sociedad, Educación y Cultura. Madrid: INE.

Jonsson, Anna-Carin; Beach, Dennis; Korp, Helena; Erlandson, Peter (2012). "Teachers' implicit theories of intelligence: influences from different disciplines and scientific theories». European Journal of Teacher Education, 35 (4), 387-400. $<$ https://doi.org/10.1080/02619768.2012.662636>.

Korp, Helena (2011). «What counts as being smart around here? The performance of smartness and masculinity in vocational upper secondary education». Education, Citizenship and Social Justice, 6 (1), 21-37. <https://doi.org/10.1177/1746197910397909>. 
Lappalainen, Sirpa; Mietola, Reetta; Lahelma, Elina (2012). «Gendered Divisions on Classed Routes to Vocational Education». Gender and Education, 2, 189-205. <https://doi.org/10.1080/09540253.2012.740445>.

Liou, Daniel D.; Rotheram-Fuller, Erinn (2019). «Where Is the Real Reform? African American Students and Their Schools Expectations for Academic Performance». Urban Education, 54 (3), 397-429. $<$ https://doi.org/10.1177/0042085915623340>.

MEFP (Ministerio de Educación y Formación Profesional) (2018). Datos y Cifras. Curso Escolar 2018/2019. Madrid: Ministerio de Educación y Formación Profesional.

Nash, Roy (2005). «Cognitive habitus and collective intelligence: concepts for the explanation of inequality of educational opportunity». Journal of Education Policy, 20 (1), 3-21. $<$ https://doi.org/10.1080/0268093042000322801>.

Nieto, Sonia; Bode, Patty (2007). Affirming diversity: The sociopolitical context of multi-cultural education, 5th ed. Boston (MA): Allyn y Bacon.

Nylund, Mattias; Rosvall, Per-Åke. (2016). «A curriculum tailored for workers? Knowledge organization and possible transitions in Swedish VET». Journal of Curriculum Studies, 48 (5), 692-710. $<$ https://doi.org/10.1080/00220272.2016.1138325>.

Nylund, Mattias; Rosvall, Per-Åke; Ledman, Kristina (2017). «The vocational-academic divide in neoliberal upper secondary curricula: the Swedish case». Journal of Education Policy, 32 (6), 788-808. <https://doi.org/10.1080/02680939.2017.1318455>.

OAKES, Jeannie (1982). "The reproduction of inequity: The content of secondary school tracking». The Urban Review, 14 (2), 107-120. <https://doi.org/10.1007/BF02174647>.

- (1985). Keeping Track: how schools structure inequality. New Haven: Yale University Press.

OAKes, Jeannie; Wells, Amy Stuart; Jones, Makeba; Datnow, Amanda (1997). "Detracking: The Social Construction of Ability, Cultural Politics, and Resistance to Reform». Teachers College Record, 98 (3), 482-510.

PÀmies, Jordi (2013). «El impacto de los agrupamientos escolares. Los espacios de aprendizaje y sociabilidad de los jóvenes de origen marroquí en Barcelona». Revista de Educación, 362, 133-158.

DOI: $<10.4438 / 1988-592 X-R E-2011-362-156>$.

Pfeffer, Fabian T. (2008). «Persistent Inequality in Educational Attainment and its Institutional Context». European Sociological Review, 24 (5), 543-565. <https://doi.org/10.1093/esr/jcn026>.

ReaY, Diane (2010). «Identity Making in Schools and Classrooms». En: Wetherell, Margaret; Mohanty, Chandra T. (eds.). The SAGE handbook of identities. Londres: Sage Publications.

Rist, Ray C. (1970). «Student Social Class and Teacher Prophecy in Ghetto Education». Harvard Educational Review, 40 (3), 411-451.

Shavit, Yossi; Müller, Walter (2000). «Vocational Secondary Education, Tracking, and Social Stratification». En: Hallinan, Maureen T. (ed.). Handbook of the Sociology of Education. Boston (MA): Springer.

Sternberg, Robert J. (2007). "Who Are the Bright Children? The Cultural Context of Being and Acting Intelligent». Educational Researcher, 36 (3), 148-155. <https:/doi.org/10.3102/0013189X07299881>. 
Sternberg, Robert J.; Davidson, Janet E. (1986). «Conceptions of giftedness: a map of the terrain». En: Sternberg, Robert J.; Davidson, Janet E. (eds.). Conceptions of giftedness. Cambridge: Cambridge University Press.

Sue, Derald W.; Sue, David (2007). Counseling the culturally diverse: Theory and practice. Nueva York (NY): John Wiley.

Tarabini, Aina; Ingram, Nicola (eds.) (2018). Educational Choices, Transitions and Aspirations in Europe: Systemic, Institutional and Subjective Challenges. Routledge.

TARABInI, Aina; Jacovkis, Judith (2019). «¿Qué conocimiento para quién? Itinerarios escolares, distribución del conocimiento y justicia escolar». Revista e-Curriculum, 17 (3), 880-908.

<https://doi.org/10.23925/1809-3876.2019v17i3p880-908>.

- (2020, en prensa). "Las transiciones a la educación secundaria postobligatoria en Cataluña: un análisis desde la sociología de la política educativa». Archivos Analíticos de Política Educativa.

Valenzuela, Angela (1999). Subtractive schooling: US-Mexican youth and the politics of schooling. Albany: State University of New York Press.

Wheelahan, Leesa (2007). «How Competency-based Training Locks the Working Class out of Powerful Knowledge: A Modified Bernsteinian Analysis». British Journal of Sociology of Education, 28 (5), 637-51. <https://doi.org/10.1080/01425690701505540>.

Youdell, Deborah (2006). Impossible Bodies, Impossible Selves: Exclusions and Student Subjectivities. Dordrecht: Springer.

Young, Michael F. D. (1971). Knowledge and control: new direction for the sociology of education. Londres: Collier Macmillan.

- (2016). "Conceptualising Vocational Knowledge: Some Theoretical Considerations». En: Young, Michael; Gamble, Jeanne. Knowledge, curriculum and qualifications for South African Further Education. Ciudad del Cabo: HSRC Press. 